\title{
Erratum to: Oxygen supply in disposable shake-flasks: prediction of oxygen transfer rate, oxygen saturation and maximum cell concentration during aerobic growth
}

\author{
Sarah Schiefelbein • Alexander Fröhlich • \\ Gernot T. John • Falco Beutler • \\ Christoph Wittmann · Judith Becker
}

Published online: 21 June 2013

(C) Springer Science+Business Media Dordrecht 2013

\section{Erratum to: Biotechnol Lett \\ DOI 10.1007/s10529-013-1203-9}

The authors would like to correct errors in the original publication of the online article.

Authors' corrections are:

In the first two pages of the original article, List of variables should be as below:

n Shaking frequency (rpm)

qo2 Specific oxygen uptake rate $\left(\mathrm{mmol} \mathrm{g}_{\mathrm{CDW}}{ }^{-1} \mathrm{~h}^{-1}\right)$
In the original publication of the article, the Eq. 2 has been published incorrectly. The correct form of Eq. 2 is provided below:

$k_{L} a=a \cdot e^{-0.5\left(\left(\frac{n-x_{0}}{b}\right)^{2}+\left(\frac{V_{\max }-y_{0}}{c}\right)^{2}\right)}$

In the online publication, the word "Supplementary Fig. 1" has been published incorrectly. The corrected form is "Supplementary file" as the supplement is NOT a Figure!
The online version of the original article can be found under doi:10.1007/s10529-013-1203-9.

Present Address:

S. Schiefelbein · A. Fröhlich · C. Wittmann ·

J. Becker $(\square)$

Institute of Biochemical Engineering, Technische

Universität Braunschweig, Gaußstraße 17, 38106

Braunschweig, Germany

e-mail: ju.becker@tu-braunschweig.de

S. Schiefelbein

e-mail: s.schiefelbein@tu-braunschweig.de

A. Fröhlich

e-mail: al.froehlich@tu-braunschweig.de

\author{
C. Wittmann \\ e-mail: c.wittmann@tu-braunschweig.de \\ G. T. John · F. Beutler \\ PreSens Precision Sensing GmbH, Regensburg, Germany \\ e-mail: g.john@presens.de \\ F. Beutler \\ e-mail: falco.beutler@PreSens.de
}

